\title{
Corpus callosum macro and microstructure in late-life depression
}

\author{
Louise Emsell $^{\star 1,2}$, Christopher Adamson ${ }^{3}$, François-Laurent De Winter ${ }^{1}$, Thibo Billiet $^{2}$, Daan
} Christiaens $^{4,5}$, Filip Bouckaert ${ }^{1,6}$, Katarzyna Adamczuk ${ }^{, 7,8}$, Rik Vandenberghe ${ }^{7}$, Marc L Seal ${ }^{3,9}$, Pascal Sienaert ${ }^{6}$, Stefan Sunaert ${ }^{2}$, Mathieu Vandenbulcke ${ }^{1}$

1. Old Age Psychiatry, University Psychiatric Centre (UPC) - KU Leuven, Belgium, ${ }^{2}$ Translational MRI \& Radiology, KU Leuven \& University Hospital Leuven, Belgium, ${ }^{3 .}$ Developmental Imaging, Murdoch Children's Research Institute, Victoria, Australia, ${ }^{4}$. Department of Electrical Engineering (ESAT), Processing of Speech and Images (PSI), Medical Image Computing, KU Leuven \& Medical Imaging Research Center, University Hospital Leuven, Belgium, ${ }^{5}$ Division of Imaging Sciences and Biomedical Engineering, Kings College London, UK, ${ }^{6} \mathrm{KU}$ Leuven, University Psychiatric Center KU Leuven, Academic center for ECT and Neurostimulation (AcCENT), Kortenberg, Belgium, ${ }^{7}$ Laboratory for Cognitive Neurology \& Department of Neurology KU Leuven, \& University Hospital Leuven, Belgium, ${ }^{8}$ Department of Neurology, Massachusetts General Hospital and Harvard Medical School, Boston, USA, ${ }^{9}$ Department of Paediatrics, The University of Melbourne, Victoria,Australia

${ }^{*}$ Corresponding author:

Dr Louise Emsell, PhD

Translational MRI

Medical Imaging Research Center

UZ Gasthuisberg, Herestraat 49 - box 7003

3000 Leuven

Belgium

louise.emsell@kuleuven.be

Abstract word count: 246

Article body word count: 4718

No. Tables: 2

No. Figures: 2

No. supplementary material: 0 


\begin{abstract}
Background: Differences in corpus callosum (CC) morphology and microstructure have been implicated in late-life depression and may distinguish between late and early-onset forms of the illness. However, a multimodal approach using complementary imaging techniques is required to disentangle microstructural alterations from macrostructural partial volume effects.
\end{abstract}

Methods: 107 older adults were assessed: 55 currently-depressed patients without dementia and 52 controls without cognitive impairment. We investigated group differences and clinical associations in 7 sub-regions of the mid-sagittal corpus callosum using T1 anatomical data, white matter hyperintensity (WMH) quantification and two different diffusion MRI (dMRI) models (multi-tissue constrained spherical deconvolution, yielding apparent fibre density, AFD; and diffusion tensor imaging, yielding fractional anisotropy, FA and radial diffusivity, $\mathrm{RD})$.

Results: Callosal AFD was lower in patients compared to controls. There were no group differences in CC thickness, surface area, FA, RD, nor whole brain or WMH volume. Lateonset of depression was associated with lower FA, higher RD and lower AFD. There were no associations between any imaging measures and psychotic features or depression severity as assessed by the geriatric depression scale. WMH volume was associated with lower FA and AFD, and higher RD in patients.

Limitations: Patients were predominantly treatment-resistant. Measurements were limited to the mid-sagittal CC. dMRI analysis was performed on a smaller cohort, $n=77$. AFD was derived from low b-value data.

Conclusions: Callosal structure is largely preserved in LLD. WMH burden may impact on CC microstructure in late-onset depression suggesting vascular pathology has additional deleterious effects in these patients.

Keywords: Depression; Late-life; Corpus callosum; White matter; MRI; Diffusion 


\section{Introduction}

Late-life depression (LLD) ${ }^{1}$ is a common, debilitating, and increasingly important disorder in the context of global ageing (Ferrari et al., 2013; Garcia-Pena et al., 2013). Its aetiology is poorly understood, in part due to the heterogeneous character of the illness. For example, LLD encompasses both patients whose depression continues from earlier adulthood (early onset - EOD) and those with a first episode after the age of 55-60 years old (late-onset, LOD)(Alexopoulos et al., 1988). This distinction is associated with differences in clinical presentation, such as a more severe course and family history in EOD, compared to greater medical comorbidity in LOD(Naismith et al., 2012). Regardless of age of onset, LLD is frequently associated with differential treatment response compared to younger patients(Aizenstein et al., 2014) and more pronounced age-related changes such as impaired executive function (e.g. in memory and processing speed)(Mackin et al., 2014), atrophy and increased cardiovascular burden(Aizenstein et al., 2014). Magnetic resonance imaging (MRI) assessment of the latter has consistently found that LLD patients have increased white matter hyperintensities (WMH)(Wang et al., 2014) which are assumed to reflect a spectrum of neuropathological changes associated with small vessel disease(Wardlaw et al., 2015). Both vascular burden and WM changes may be central to LLD pathology (Alexopoulos et al., 1997; Naismith et al., 2012; Valkanova and Ebmeier, 2013; Wen et al., 2014) thus motivating further characterisation of WM structural alterations in the illness using multimodal MRI measures.

\section{The corpus callosum and late-life depression}

The CC comprises more than a hundred million topographically arranged axons and forms the greatest inter-hemispheric WM network in the human brain. As such, it forms a critical component of brain function, supporting higher-order processing throughout the entire cortex, from prefrontal systems through to the visual system (Schmahmann J and Pandya D, 2006;

\footnotetext{
1 Abbreviations: AFD: Apparent fibre density; CC: corpus callosum; CSD: constrained spherical deconvolution; CSF: cerebrospinal fluid; DTI: diffusion tensor imaging; EOD: early-onset depression; FA: fractional anisotropy; FOD: Fiber orientation distribution; LLD: late-life depression; LOD: late-onset depression; MT-CSD: multi-tissue constrained spherical deconvolution RD: radial diffusivity; sMRI: structural magnetic resonance imaging; WM: white matter; $\mathrm{WMH}$ : white matter hyperintensities
} 
Tomasch, 1954). It is hypothesised that the calibre and density of callosal axons reflect the functional capacity of the brain regions they connect and thus the tissue composition of the CC is heterogenous to reflect functional specialisation(Aboitiz et al., 1992). Given the functional anatomy of the $\mathrm{CC}$, regional structural changes in the $\mathrm{CC}$ might therefore contribute to the impaired cognition, emotional regulation and psychomotor dysfunction that characterises depression.

Indeed, there is some evidence to suggest $\mathrm{CC}$ changes may reflect both trait abnormalities of depression(Yamada et al., 2015), be mood-state dependent(van Uden et al., 2015; Walterfang et al., 2009) or reflect specific features of the illness such as psychosis(Sarrazin et al., 2015) and the time of disease-onset(Kemp et al., 2013). The latter is of most relevance to our study of LLD patients and several studies deserve highlighting. In a 10-year follow-up study, Cyprien et al(Cyprien et al., 2014) found a smaller CC to be predictive of incident depression in a 10-year period in women, but not men. Hahn et al (Hahn et al., 2014) investigated callosal subregions in LOD patients and found a smaller anterior and posterior CC in patients, which correlated with cognitive measures, but not depression severity or illness duration. Similarly, in a morphometry study including both EOD and LOD patients, Ballmaier et al (Ballmaier et al., 2008) found callosal thinning in both the genu and splenium, which correlated with memory and attention functioning only in the LOD group. Notably, the LOD group also had a thinner CC compared to the EOD group suggesting structural differences could discriminate between LLD subtypes. Studies applying diffusion tensor imaging (DTI) report mixed results with both no group-level differences (or not surviving multiple comparisons correction) in CC microstructure in patients (Colloby et al., 2011; Nobuhara et al., 2006) as well as localised differences in the CC (Mettenburg et al., 2012; Reppermund et al., 2014). Interestingly, Alexopoulus et al (Alexopoulos et al., 2008) related CC deficits to poor treatment response in LLD.

The role of multi-modal MRI to investigate brain macro- and microstructure What differentiates our study from previous investigations and which adds to new knowledge about LLD, is the combined analysis of both T1-weighted structural MRI (sMRI) data and 
diffusion MRI (dMRI) data using two different modelling approaches. Because WM appears homogeneous on T1-weighted scans, it is not possible to reliably perform region-of-interest (ROI) studies specific to most WM fibre bundles, therefore most studies investigate either regional morphometric analysis of SMRI data, or regional microstructural analysis of diffusion MRI data. When studies do apply both modalities they typically perform a voxel-based analysis (VBA) (including tract-based spatial statistics, TBSS) of both data types and compare the results e.g.(Mettenburg et al., 2012; Sexton et al., 2012a). However, by doing so, such studies cannot assess the relationship between macro and microstructural features in exactly the same regions (e.g. they are confounded by differences associated with image processing). This is particularly important in dMRI analysis, where partial volume contamination can significantly confound the estimated parameters(Alexander et al., 2001; Raffelt et al., 2012; Vos et al., 2011). Combining complementary sMRI and dMRI measures in the same well-defined region enables a more complete understanding of structural changes at different length scales.

\section{Two diffusion MRI models}

Presently, the most widely used dMRI measures are FA, mean (MD), radial (RD) and axial diffusivity (AD), derived from the simple 6-parameter diffusion tensor imaging (DTI) model(Basser et al., 1994). In this approach, each voxel is assigned a unique degree of unidirectional anisotropy that is assumed to reflect the dominant underlying axonal architecture. DTI metrics such as FA reflect local changes in bundle composition, which may be caused by either microstructural alterations such as axon loss or reduced membrane integrity, or architectural changes such as increased axon dispersion and the presence of multiple fibre populations(Basser and Pierpaoli, 1996; Concha, 2014). In contrast to DTI, constrained spherical deconvolution (CSD) assumes constant anisotropy along fibres(Tournier et al., 2007). This means that CSD and DTI offer alternative and complementary methods to investigate WM, with CSD improving upon the tensor model by being more directly related to the measured diffusion-weighted signal and being more fibre bundle specific(Tournier et al., 2008). For example, CSD yields the fibre orientation distribution function (FOD) from which parameters relating to partial volume fractions (the relative space in the voxel attributed to a 
certain compartment) can be derived, and that are assumed to reflect fibre density. The "apparent fibre density" (AFD) measure(Raffelt et al., 2012) has been shown to correlate well with histological descriptions, and has successfully been applied in a number of studies examining WM abnormalities in clinical populations (Farquharson et al., 2014; Raffelt et al., 2012; Scheck et al., 2015; Vaughan et al., 2013), but not yet to study depression.

\section{Study aims and hypotheses}

In this study, our aims were threefold: to identify (1) potential global and regional structural differences in the CC associated with LLD, (2) any association between macro and microstructural differences and white matter lesion burden and (3) any associations between structural differences, depression sub-types and depressive symptomatology. We hypothesised that depression would be associated with regional callosal thinning and a reduced callosal area in anterior and posterior divisions of the CC, with reduced FA, increased RD and decreased AFD mirroring these changes. Moreover we hypothesised that CC structure would be adversely affected by increasing WM lesion load, depression severity and the presence of psychotic features. 


\section{Methods}

\section{Participants}

We included 55 currently depressed patients with LLD and 52 age- and gender-matched healthy control participants without current or previous depressive illness, nor cognitive impairment as assessed by formal clinical rating scales. Two additional participants were excluded prior to analysis due to incidental radiological findings of an ateriovenous malformation and frontal lobe lesion respectively. Patients were recruited from the Old Age Psychiatry Department of the University Psychiatric Centre of the University of Leuven, Belgium and control participants were recruited through an existing departmental database and advertisement in a local newspaper. Part of the control cohort $(n=46)$ were recruited as part of another study investigating cerebral amyloidosis(Adamczuk et al., 2013; Adamczuk et al., 2016). This subset fulfilled additional inclusion criteria: a clinical dementia rating score $=0$ and mini-mental state examination (MMSE) score $>26$, and were stratified for APOE $\varepsilon 4$ allele. Patients were included according to the following criteria: 1 . older than 55 years; 2. a major depressive disorder with or without psychotic features according to DSM IV criteria and confirmed by the Mini International Neuropsychiatric Interview (MINI) (Sheehan et al., 1998) 3. not primarily referred for assessment of cognitive impairment. Exclusion criteria for all participants were: 1. comorbid major psychiatric illness; 2. previous or current alcohol and/or drug dependence; 3. neurological illness, including stroke, transient ischemic attacks and dementia; 4. illness or medication precluding cognitive testing; 5. metal implants precluding MRI scanning; and 6. ECT in the last 6 months prior to inclusion. The study was approved by the University Hospitals Leuven ethical committee, and all participants gave their written informed consent prior to the study in accordance with the Declaration of Helsinki. Full details of participant characteristics are summarised in Table 1.

\section{Structured assessment of mood and cognition}

All assessments were performed using validated Dutch versions of each rating scale. The diagnostic status of patients and controls was confirmed by Structured Clinical Interview for 
DSM IV (SCID-I)(American-Psychiatric-Association, 1994). Depressive symptoms were assessed using the Geriatric Depression Scale(Yesavage et al., 1982) and MontgomeryÅsberg Depression Rating Scale (MADRS). Cognition was assessed by Mini-Mental State Examination (MMSE).

\section{Imaging}

\section{Magnetic resonance imaging}

Structural MRI, diffusion MRI (dMRI) and fluid attenuated inversion recovery (FLAIR) data were acquired using an 8-channel head coil on a $3 T$ Philips Intera scanner (Best, The Netherlands). High-resolution 3D turbo field echo (3DTFE) T1-weighted images were acquired with parameters: $T R=9.6 \mathrm{~s}, \mathrm{TE}=4.6 \mathrm{~s}$, flip angle $=8^{\circ}$, Slice thickness $=1.2 \mathrm{~mm}$, in-plane voxel-size $=0.98 \times 0.98 \times 1.2 \mathrm{~mm}^{3}, 182$ axial slices. Diffusion MRI data were acquired using an echo planar imaging sequence with diffusion-weighting, $b=800 \mathrm{~s} / \mathrm{mm}^{2}$, applied along 45 uniformly distributed gradient directions and included six non-diffusion weighted images. Constant scan parameters were TR/TE $=11000 \mathrm{~ms} / 55 \mathrm{~ms}, 68$ slices, voxel size $1.98 \times 1.98 \times$ $2.2 \mathrm{~mm}^{3}$. The 2D-FLAIR data were acquired for routine clinical assessment with the following main consistent parameters: TR: $11000 \mathrm{~ms}$, voxel size $=0.45 \mathrm{~mm} \times 0.45 \mathrm{~mm} \times 5 \mathrm{~mm}$.

Due to decommissioning of the $3 \mathrm{~T}$ Intera scanner, 19 controls were scanned with identical sequence parameters on a 3T Philips Achieva scanner using a 32-channel head coil. Due to the particular sensitivity of dMRI data to differences in scanner hardware, we excluded these 19 controls from the dMRI analysis.

\section{White matter hyperintensities and total brain volume}

The automated quantitative assessment of white matter lesion load and total brain volume was performed using MSMetrix (Jain et al 2015). This approach combines T1 and FLAIR data in a probabilistic model in which lesions are detected as outliers when segmenting brain tissue into grey matter, WM and CSF compartments (Jain et al, 2015). Total brain volume was obtained by summing the respective brain tissue fractions. 


\section{Corpus callosum morphology}

CC thickness and area were obtained using an automated pipeline(Adamson et al., 2014). Firstly, the mid-sagittal plane was identified using acpcdetect(Ardekani and Bachman, 2009) in combination with FSL-FLIRT registration tools(Jenkinson and Smith, 2001). Automated segmentation of the CC followed an initialisation step aligning each subjects' dataset to template probability maps constructed from the OASIS dataset(Marcus et al., 2007). Datadriven subsequent refinement was achieved using mathematical morphological operations and a post-processing step to remove posterior and pericallosal vessels. Thickness profiles were generated using streamlines generated from a solution to Laplace's equation in callosal voxels, determined to form non-overlapping, cross-sectional contour lengths(Adamson et al., 2011). Additionally, the CC was divided into eight sections based on the combination of two parcellation schemes: the Witelson scheme, based on geometric proportions(Witelson, 1989) and the Hofer-Frahm scheme which is based on DTI data(Hofer and Frahm, 2006). These regions included: rostrum, genu, anterior body, mid-body, posterior body, isthmus, dorsal splenium and ventral splenium (See Figure 1).

\section{Corpus callosum microstructure}

\section{Diffusion tensor imaging}

One control and 10 patients were excluded from the dMRI analysis due to absent or poor quality data due to motion artefacts. After correction for subject motion and geometric distortion(Leemans and Jones, 2009), the DTI parameters FA and RD were estimated using a normalised linear least squares approach implemented in ExploreDTI(Leemans et al., 2009). Each subjects' corrected DTI parameter map was diffeomorphically registered to their corresponding T1-image using ANTS(Avants et al., 2008) based on cross-correlation between the T1 WM segmentation and FA map, and mutual information between the T1 image and average DWI. The warping parameters were then applied to the CC segmentations, from which the mean FA and RD were extracted. 
Apparent fibre density

Measures of apparent fibre density (Raffelt et al., 2012) were computed in all subjects with multi-tissue constrained spherical deconvolution (MT-CSD)(Jeurissen et al., 2014). MT-CSD incorporates information from different tissue types extracted directly from the dMRI data to improve the precision of the FOD estimation. MT-CSD is typically used with a 3-tissue model for WM, GM, and CSF in data with at least 2 shells (+ the $b=0$ image). In this study, because our data is single-shell, we used MT-CSD to reduce partial volume effects at the WM-CSF interface. First, all data were corrected for intensity inhomogeneity. The bias field was estimated in the $b=0$ image using FSL-FAST(Zhang et al., 2001) and its inverse was subsequently applied on all volumes in the DWI series. Secondly, response functions of single-fibre WM and isotropic, ventricular CSF were estimated from the DWI data in predefined masks, and later averaged across all healthy controls. The single-fibre WM mask was defined as all voxels within a brain mask in which fractional anisotropy $(F A)>0.7$. A ventricular CSF mask was obtained by first thresholding FA $<0.1$, and then applying connected component analysis to the resulting segmentation. The second largest component corresponded to the ventricle mask. Finally, the mean WM and CSF response functions were used in MT-CSD to reconstruct fibre orientation distributions (FOD) in all patients and controls. In single fibre voxels, such as in the corpus callosum, AFD equals the isotropic integral of the FOD, without requiring fixel segmentation(Raffelt et al., 2015).

The resulting AFD maps were intensity-normalized across subjects by rescaling with the median $b=0$ intensity in a full-brain mask and coregistered with the $T_{1}$-weighted image. Bias field correction and intensity normalisation are needed for AFD analysis because, in contrast to the diffusion tensor which is normalised to $b=0, C S D$ is sensitive to (scales linearly with) the DWI image intensity. Finally, AFD was averaged in each region of the corpus callosum parcellation in T1 space in the same way as FA and RD, described above.

\section{Statistical analysis}

Group differences in corpus callosum macro and microstructure

All statistics were performed using IBM SPSS Statistics for Macintosh, Version 23.0. Two MANCOVA analyses were used to identify group differences in (1) corpus callosum surface 
area and median thickness across the entire mid-sagtital slice (hereafter referred to as "whole CC" measures), (2) FA, RD and AFD. In both cases, diagnosis was a fixed factor, and age and total brain volume were covariates of no-interest. Additionally, potential scanner effects on the T1-weighted data were accounted for by including scanner as a fixed factor in the thickness and area analyses. By definition, WMH influence diffusion measures, which are sensitive to T2 signal changes, therefore whole brain $\mathrm{WMH}$ volume was included as a covariate in the dMRI analyses. To illustrate the effect of $\mathrm{WMH}$ volume on the whole CC measures, Pearson's correlations were performed.

A further series of 5 MANCOVAs were used to assess regional differences between patients and controls. In these analyses, each region was included as a dependent variable in the same model, and the fixed factors and covariates were the same as in the whole mid-sagittal corpus callosum MANCOVAs. Following quality control, the small size of the rostrum in many participants made parameter estimates unreliable in this region and it was therefore excluded from all analyses. To control for multiple comparisons, SPSS Bonferroni adjusted p-values of $<0.05$ obtained in each separate MANCOVA were further corrected to account for the number of MANCOVAS i.e. $p$-values were deemed statistically significant below $p<0.01(\alpha=0.05 / 5)$.

\section{Post hoc clinical associations in patients}

A series of exploratory ANCOVAs were used to investigate potential relationships between whole CC measures, regional group differences and clinically important factors in patients. These variables included: presence of psychosis, late (>55 years) or early onset of depression and GDS score. In all models, age, total brain volume and WMH volume were included as covariates. All patients were scanned on the same scanner. SPSS Bonferroni adjusted $p$-values of $<0.05$ were corrected further to account for the three factors of interest i.e. $p<0.017(\alpha=0.05 / 3)$ was considered statistically significant. On account of the exploratory nature of these analyses, correction for 15 comparisons ( 5 metrics $\times 3$ clinical factors) was deemed overly conservative. 


\section{Results}

Structural differences in the whole corpus callosum

The median AFD across the whole mid-sagittal corpus callosum was reduced in patients compared to controls $\left(F(1,73)=4.307, p=0.042, \eta^{2}=0.057\right)$. We did not detect any statistically significant group differences in whole mid-sagittal callosal thickness or area, nor differences in any DTI measure between patients and controls. Whilst there were no differences in total WMH volume between patients and controls, in either the whole cohort $(t(104)=-0.983$, $p=0.328)$ nor $d M R I$ sub-sample $(t(75)=-0.383, p=0.703)$, WMH volume was associated with both macrostructural and diffusion changes in patients only. Specifically, increasing lesion load was associated with reduced area $(\rho=-0.292, p=0.036)$, thickness $(\rho=-0.327, p=0.018)$, FA $(\rho=-0.375, p=0.011)$ and AFD $(\rho=-0.418, p=0.004$, and higher $\operatorname{RD}(\rho=0.523, p<0.001)$.

\section{Regional differences}

In line with the global findings, we detected regional differences in AFD in the CC body and ventral splenium (Figure 2). However, these did not survive the additional multiple comparisons correction. There were no other regional group differences in structural or DTI measures.

The complete results are summarised in Table 2.

Post hoc clinical associations in patients

There was a main effect of LOD on all dMRI measures, but not macrostructural measures. LOD was associated with lower $F A\left(F(1,34)=8.380, p=0.007, \eta^{2}=1.98\right)$, higher RD $\left(F(1,34)=6.468, p=0.016, \eta^{2}=1.60\right)$ and lower AFD $\left(F(1,34)=4.579, p=0.040, \eta^{2}=0.119\right)$. The effect of FA survived multiple comparisons correction. Further analysis revealed that this could not be explained by differences in WMH volume between LOD and EOD patients $(t(44)=-0.070, p=0.944)$. The effect of LOD on FA was regional and confined to the body of the $\mathrm{CC}$ (anterior body: $\mathrm{F}(1,38)=4.931, \mathrm{p}=0.033, \eta^{2}=0.127$, mid-body: $\mathrm{F}(1,38)=9.787, \mathrm{p}=0.004$, 
$\eta^{2}=0.224$, posterior body: $\left.F(1,38)=6.011, p=0.020, \eta^{2}=0.150\right)$. There was a borderline effect on the genu: $F(1,38)=3.934, p=0.055, \eta^{2}=0.104$. There were no associations between any imaging measures and the presence of psychotic features or depression severity (GDS). 


\section{Discussion}

In this study we found that CC macrostructure was preserved in LLD. There was some evidence to suggest that microstructure was altered throughout the $\mathrm{CC}$, and that in our study AFD was more sensitive than DTI metrics for detecting these differences. Moreover dMRI measures appeared to discriminate between late and early-onset depression, whilst no association was found between CC structure and psychosis and depression severity.

These results are largely consistent with the extant literature, as the majority of studies have found extra-callosal WM changes in LLD, particularly in prefrontal regions and temporal cortex (Mettenburg et al., 2012; Nobuhara et al., 2006; Reppermund et al., 2014; Sexton et al., 2012a). Interestingly, this is in contrast to studies including patients with bipolar disorder (BD), which is also characterised by an inherently depressive profile, yet frequently associated with callosal abnormalities(Emsell et al., 2013; Sarrazin et al., 2015). Notably, a small DTI study by Sexton et al (Sexton et al., 2012b) found that late-life BD $(n=10)$ could be discriminated from unipolar LLD $(n=36)$ on the basis of lower FA in the BD group. One might hypothesise that other features of BD such as psychosis may underlie CC changes, as CC structural deficits have been linked with a broad spectrum of psychotic disorders(Francis et al., 2016). In our study however, psychosis $(n=19)$ was unrelated to CC structure. This could be due to the relatively small sub-sample or reflect different disease mechanisms across the mood disorder spectrum.

In the context of heterogeneity within depression subtypes, it is also noteworthy that our lateonset patients had more pronounced microstructural changes than the early-onset group. This too is in agreement with other studies, including Ballmaier et al (Ballmaier et al., 2008) who reported more extensive callosal thinning in LOD than EOD compared to controls, and Sexton et al (Sexton et al., 2012a) who reported more widespread FA reductions in LOD compared to controls. In contrast to our study of currently depressed patients, Sexton et al did find DTI differences in the corpus callosum between patients and controls, which could be due to either differences in the clinical sample or DTI processing (e.g. group TBSS v native space ROI). There were however similarities between our respective findings, for example, no 
group differences in total WM lesion load or CC volume (based on FSL-VBM of partial-volume maps).

More pronounced age-associated microstructural changes in callosal white matter may render older subjects more vulnerable for depression due to a decrease of cognitive resources involved in problem solving strategies. Indeed, aging has been associated with increased reliance on interhemispheric communication serving a compensatory role in cognitive performance (Cabeza et al., 2002). Accordingly, callosal microstructural impairment in older adults is associated with decreased processing speed and working memory performance (Kennedy and Raz, 2009). Moreover, CC volume changes in LOD have been specifically associated with cognitive measures rather than mood symptoms (Hahn et al. 2014). Although extensive cognitive assessment targeting those mental operations that rely on CC integrity (Gazzaniga, 2000) was beyond the scope of the present study, such focused brain-behavior correlations should be included in future research on the role of CC microstructure in the pathogenesis of LOD.

\section{The role of WMH in LLD}

Increased rates of WMH are frequently associated with depression and may be more severe in LOD(Herrmann et al., 2008). However, this was not the case in our study. Nevertheless, whilst we did not detect a difference in total $\mathrm{WMH}$ volume between patients and controls, we found that WMH burden was associated with reductions in FA and AFD, and increases in RD in patients only. This is in agreement with Shimony et al (Shimony et al., 2009), who excluded discrete WMH from DTI measurements. Moreover, the authors also reported regionally lower anisotropy in patients normal-appearing WM, which was more pronounced in prefrontal WM, and did not reach significance in the $\mathrm{CC}$. Whilst this suggests that diffusion changes may be observed independently of WMH, another larger TBSS study including 151 elderly participants with small vessel disease and depressive symptoms found that statistically significant widespread deficits in diffusion measures disappeared after adjustment for total WMH and lacunar infarcts(van Uden et al., 2015). Regional rather than global measures of WMH may be more helpful in understanding the link between LLD, WMH and diffusion measures. For example, some studies have localised group differences in ischaemic changes 
to the prefrontal WM tracts(Sheline et al., 2008; Thomas et al., 2002). In this context, regional disruption to WM circuitry by WMH could both impact on diffusion measures and explain disease related effects in the absence of group differences in total WMH volume.

\section{The relationship between different diffusion measures and microstructure}

It is clear that the relationship between different imaging parameters and the pathophysiology of LLD is dynamic and complex. In our study, we applied two different types of dMRI analysis based on the same raw dMRI data, which yielded apparently inconsistent results i.e. no group differences in anisotropy or radial diffusivity, yet differences in apparent fibre density. This highlights the challenges associated with linking disease-related effects on microstructure with specific dMRI measures. In our case, we believe the increased sensitivity of AFD compared to DTI was due to differences in the way we controlled partial volume effects. In contrast to the DTI analysis, in the AFD analysis, we used a two-tissue model (WM-CSF) to restrict the measurement to callosal WM and mitigate the effects of CSF contamination from the lateral ventricles. However, as we did not observe any macrostructural differences in the CC (i.e. in thickness or area), we cannot draw any conclusions about the hypothesised partial volume bias on DTI measures. Notably, our estimation of AFD was based on relatively low bvalue data $800 \mathrm{~mm}^{2} / \mathrm{s}$, which means out findings may not generalise to other study conditions using higher b-value data (e.g. $3000 \mathrm{~mm}^{2} / \mathrm{s}$ ). Whilst our two-tissue approach may have enhanced the sensitivity of the AFD analysis, it could have also increased the influence of intensity bias field correction and normalisation between subjects. In this context, as AFD estimation is based on calculations derived from voxels throughout the whole brain, global or regional differences in $\mathrm{T} 2$ relaxation times between groups, such as due to $\mathrm{WMH}$, could have significantly influenced the results. Future methodological work examining whole brain MTCSD and DTI derived measures coupled with regional WMH segmentation will further our understanding of these issues.

Our study involves indirect imaging measures and therefore neither attempts to nor can address pathophysiological mechanisms underlying LLD. However, with regard to a neurobiological explanation of our findings, we may not be detecting an actual reduction in 
callosal axon density as the assumptions underlying AFD are based on restricted diffusion characteristics at higher b-values (e.g. $3000 \mathrm{~mm}^{2} / \mathrm{s}$ ), whilst our data also captures the effects of hindered extracellular diffusion not fully attenuated at $b=800 \mathrm{~mm}^{2} / \mathrm{s}$. This effect of hindered extra-axonal diffusion is incorporated in the estimated single-fibre response function, and is hence implicitly assumed constant throughout the brain. When this is not the case, CSD and derived AFD metrics can become sensitive to extra-axonal changes. Nevertheless, the AFD profile we observed across the CC is largely consistent with histological (Aboitiz et al., 1992) and low q-value imaging data(Suzuki et al., 2016) describing axon diameter and density. This increases confidence in the observed differences being, at least in part, due to genuine microstructural differences affecting the number, size and/or density of callosal axons. Furthermore, the use of MT-CSD to account for partial volume contamination of adjacent ventricular CSF improved the estimation of AFD metrics in the CC by accounting for the free water compartment volume fraction. Finally, despite its less straightforward interpretation the statistically significant group differences indicate that reduced AFD in the CC may nevertheless be a suitable biomarker of late life depression.

\section{Limitations}

Our study applied a multimodal assessment of the $C C$ in a relatively large sample, and is the first to report AFD changes in depression. Nevertheless, there are some limitations that should be noted. 1) The dMRI and patient sub-group analyses contained fewer participants than the total sample and should be interpreted with due caution. Notably, our positive findings were only present when applying more complex ANCOVA models rather than t-tests, which could be interpreted in terms of a lack of power, the subtlety of the effects, and as evidence of the complexity of age-related and disease-related interactions in older adults. 2) We could not address medication status in our analysis as our study was not designed to do so. Notably, our patient sample comprised primarily of treatment resistant participants, who may represent a biologically distinct subgroup. 3) We used the MMSE to assess cognitive function. Whilst this is a useful clinical measure, it is less well suited for investigating detailed aspects of cognition, and therefore we were unable to relate our imaging findings to cognitive performance. 4) patients were recruited from an academic referral centre for LLD, which 
could form a selection bias towards more severe, therapy resistant and psychotic depressions and thus reduce the applicability of our findings to the general population. 5) We estimated AFD using a sub-optimal approach based on low b-value data, which complicates the interpretation of our findings. 6) We restricted our analysis to a single mid-sagittal slice of the $\mathrm{CC}$, therefore we cannot generalise our results to the whole structure.

\section{Conclusion}

Late-life depression is associated with subtle, microstructural changes in the corpus callosum, which are unrelated to psychosis or depression severity, and are more pronounced in patients with a late-onset of LLD. Combining anatomical and diffusion data provides greater insight into structural MRI changes than using either technique in isolation.

\section{Acknowledgements}

The authors would like to thank Dr Kristof Vansteenlandt for providing statistical advice and Dr Donald Tournier for advice regarding the AFD measurement and its interpretation. An abstract based on this work was presented at the $24^{\text {th }}$ Annual Meeting of the International Society for Magnetic Resonance Imaging in Medicine, $7^{\text {th }}-13^{\text {th }}$ May 2016 , Singapore.

Funding: This work was supported by the Foundation for Alzheimer Research (RV., SAO-

FRMA 09013, 11020, 13007), KU Leuven (RV., OT/08/056, OT/12/097), FWO (Research

Foundation Flanders; KA and MV., G.0746.09), and IWT (Innovation by Science and

Technology; DC., SB121013)

\section{References}


1. Aboitiz, F., Scheibel, A.B., Fisher, R.S., Zaidel, E., 1992. Fiber composition of the human corpus callosum. Brain Res 598, 143-153.

2. Adamczuk, K., De Weer, A.S., Nelissen, N., Chen, K., Sleegers, K., Bettens, K., Van Broeckhoven, C., Vandenbulcke, M., Thiyyagura, P., Dupont, P., Van Laere, K., Reiman, E.M., Vandenberghe, R., 2013. Polymorphism of brain derived neurotrophic factor influences beta amyloid load in cognitively intact apolipoprotein E epsilon4 carriers. Neuroimage Clin 2, 512-520.

3. Adamczuk, K., Schaeverbeke, J., Nelissen, N., Neyens, V., Vandenbulcke, M., Goffin, K., Lilja, J., Hilven, K., Dupont, P., Van Laere, K., Vandenberghe, R., 2016. Amyloid imaging in cognitively normal older adults: comparison between (18)Fflutemetamol and (11)C-Pittsburgh compound B. Eur J Nucl Med Mol Imaging 43, 142-151.

4. Adamson, C., Beare, R., Walterfang, M., Seal, M., 2014. Software Pipeline for Midsagittal Corpus Callosum Thickness Profile Processing : Automated Segmentation, Manual Editor, Thickness Profile Generator, Group-Wise Statistical Comparison and Results Display. Neuroinformatics.

5. Adamson, C.L., Wood, A.G., Chen, J., Barton, S., Reutens, D.C., Pantelis, C., Velakoulis, D., Walterfang, M., 2011. Thickness profile generation for the corpus callosum using Laplace's equation. Hum Brain Mapp 32, 2131-2140.

6. Aizenstein, H.J., Khalaf, A., Walker, S.E., Andreescu, C., 2014. Magnetic resonance imaging predictors of treatment response in late-life depression. J Geriatr Psychiatry Neurol 27, 24-32.

7. Alexander, A.L., Hasan, K.M., Lazar, M., Tsuruda, J.S., Parker, D.L., 2001. Analysis of partial volume effects in diffusion-tensor MRI. Magnetic resonance in medicine : official journal of the Society of Magnetic Resonance in Medicine / Society of Magnetic Resonance in Medicine 45, 770-780.

8. Alexopoulos, G.S., Meyers, B.S., Young, R.C., Campbell, S., Silbersweig, D., Charlson, M., 1997. 'Vascular depression' hypothesis. Arch Gen Psychiatry 54, 915922.

9. Alexopoulos, G.S., Murphy, C.F., Gunning-Dixon, F.M., Latoussakis, V., Kanellopoulos, D., Klimstra, S., Lim, K.O., Hoptman, M.J., 2008. Microstructural white matter abnormalities and remission of geriatric depression. Am J Psychiatry 165, 238-244.

10. Alexopoulos, G.S., Young, R.C., Meyers, B.S., Abrams, R.C., Shamoian, C.A., 1988. Late-onset depression. Psychiatr Clin North Am 11, 101-115.

11. American-Psychiatric-Association, 1994. DSM-IV-TR Diagnostic and Statistical Manual of Mental Disorders, 4th ed. American Psychiatric Press Inc, Arlington.

12. Ardekani, B.A., Bachman, A.H., 2009. Model-based automatic detection of the anterior and posterior commissures on MRI scans. Neuroimage 46, 677-682.

13. Avants, B.B., Epstein, C.L., Grossman, M., Gee, J.C., 2008. Symmetric diffeomorphic image registration with cross-correlation: evaluating automated labeling of elderly and neurodegenerative brain. Med Image Anal 12, 26-41.

14. Ballmaier, M., Kumar, A., Elderkin-Thompson, V., Narr, K.L., Luders, E., Thompson, P.M., Hojatkashani, C., Pham, D., Heinz, A., Toga, A.W., 2008. Mapping callosal morphology in early- and late-onset elderly depression: an index of distinct changes in cortical connectivity. Neuropsychopharmacology 33, 1528-1536. 
15. Basser, P.J., Mattiello, J., LeBihan, D., 1994. Estimation of the effective self-diffusion tensor from the NMR spin echo. J Magn Reson B 103, 247-254.

16. Basser, P.J., Pierpaoli, C., 1996. Microstructural and physiological features of tissues elucidated by quantitative-diffusion-tensor MRI. J Magn Reson B 111, 209-219.

17. Cabeza, R., Anderson, N.D., Locantore, J.K., McIntosh, A.R., 2002. Aging gracefully: compensatory brain activity in high-performing older adults. Neuroimage 17, 13941402.

18. Colloby, S.J., Firbank, M.J., Thomas, A.J., Vasudev, A., Parry, S.W., O'Brien, J.T., 2011. White matter changes in late-life depression: a diffusion tensor imaging study. J Affect Disord 135, 216-220.

19. Concha, L., 2014. A macroscopic view of microstructure: using diffusion-weighted images to infer damage, repair, and plasticity of white matter. Neuroscience $276,14-$ 28.

20. Cyprien, F., Courtet, P., Poulain, V., Maller, J., Meslin, C., Bonafe, A., Le Bars, E., Ancelin, M.L., Ritchie, K., Artero, S., 2014. Corpus callosum size may predict late-life depression in women: a 10-year follow-up study. J Affect Disord 165, 16-23.

21. Emsell, L., Leemans, A., Langan, C., Van Hecke, W., Barker, G.J., McCarthy, P., Jeurissen, B., Sijbers, J., Sunaert, S., Cannon, D.M., McDonald, C., 2013. Limbic and callosal white matter changes in euthymic bipolar I disorder: an advanced diffusion magnetic resonance imaging tractography study. Biol Psychiatry 73, 194-201.

22. Farquharson, S., Raffelt, D., Sadeghian, F., Tournier, J.D., Mandelstam, S., Schneider-Kolsky, M., 2014. Apparent fibre density (AFD) analysis reveals decreases in axonal density in the white matter pathways of patients with grey matter heterotopia, 22nd Annual Meeting of International Society for Magnetic Resonance Imaging in Medicine Milan, Italy.

23. Ferrari, A.J., Charlson, F.J., Norman, R.E., Flaxman, A.D., Patten, S.B., Vos, T., Whiteford, H.A., 2013. The epidemiological modelling of major depressive disorder: application for the Global Burden of Disease Study 2010. PLoS One 8, e69637.

24. Francis, A.N., Mothi, S.S., Mathew, I.T., Tandon, N., Clementz, B., Pearlson, G.D., Sweeney, J.A., Tamminga, C.A., Keshavan, M.S., 2016. Callosal Abnormalities Across the Psychosis Dimension: Bipolar Schizophrenia Network on Intermediate Phenotypes. Biol Psychiatry.

25. Garcia-Pena, C., Wagner, F.A., Sanchez-Garcia, S., Espinel-Bermudez, C., JuarezCedillo, T., Perez-Zepeda, M., Arango-Lopera, V., Franco-Marina, F., RamirezAldana, R., Gallo, J.J., 2013. Late-life depressive symptoms: prediction models of change. J Affect Disord 150, 886-894.

26. Gazzaniga, M.S., 2000. Cerebral specialization and interhemispheric communication: does the corpus callosum enable the human condition? Brain 123 ( Pt 7), 1293-1326.

27. Hahn, C., Lim, H.K., Won, W.Y., Joo, S.H., Ahn, K.J., Jung, W.S., Lee, C.U., 2014. Sub-regional volumes changes of the corpus callosum in the drug naive patients with late-onset depression. Prog Neuropsychopharmacol Biol Psychiatry 56C, 46-51.

28. Herrmann, L.L., Le Masurier, M., Ebmeier, K.P., 2008. White matter hyperintensities in late life depression: a systematic review. J Neurol Neurosurg Psychiatry 79, 619624. 
29. Hofer, S., Frahm, J., 2006. Topography of the human corpus callosum revisited-comprehensive fiber tractography using diffusion tensor magnetic resonance imaging. Neuroimage 32, 989-994.

30. Jenkinson, M., Smith, S., 2001. A global optimisation method for robust affine registration of brain images. Med Image Anal 5, 143-156.

31. Jeurissen, B., Tournier, J.D., Dhollander, T., Connelly, A., Sijbers, J., 2014. Multitissue constrained spherical deconvolution for improved analysis of multi-shell diffusion MRI data. Neuroimage 103, 411-426.

32. Kemp, A., MacMaster, F.P., Jaworska, N., Yang, X.R., Pradhan, S., Mahnke, D., Courtright, A., Ramasubbu, R., 2013. Age of onset and corpus callosal morphology in major depression. J Affect Disord 150, 703-706.

33. Kennedy, K.M., Raz, N., 2009. Aging white matter and cognition: differential effects of regional variations in diffusion properties on memory, executive functions, and speed. Neuropsychologia 47, 916-927.

34. Leemans, A., Jeurissen, B., Sijbers, J., Jones, D., 2009. ExploreDTI: a graphical toolbox for processing, analyzing, and visualizing diffusion MR data, 17th Annual Meeting of Intl Soc Mag Reson Med, Hawaii, USA.

35. Leemans, A., Jones, D.K., 2009. The B-matrix must be rotated when correcting for subject motion in DTI data. Magn Reson Med 61, 1336-1349.

36. Mackin, R.S., Nelson, J.C., Delucchi, K.L., Raue, P.J., Satre, D.D., Kiosses, D.N., Alexopoulos, G.S., Arean, P.A., 2014. Association of age at depression onset with cognitive functioning in individuals with late-life depression and executive dysfunction. Am J Geriatr Psychiatry 22, 1633-1641.

37. Marcus, D.S., Wang, T.H., Parker, J., Csernansky, J.G., Morris, J.C., Buckner, R.L., 2007. Open Access Series of Imaging Studies (OASIS): cross-sectional MRI data in young, middle aged, nondemented, and demented older adults. J Cogn Neurosci 19, 1498-1507.

38. Mettenburg, J.M., Benzinger, T.L., Shimony, J.S., Snyder, A.Z., Sheline, Y.I., 2012. Diminished performance on neuropsychological testing in late life depression is correlated with microstructural white matter abnormalities. Neuroimage 60, 21822190.

39. Naismith, S.L., Norrie, L.M., Mowszowski, L., Hickie, I.B., 2012. The neurobiology of depression in later-life: clinical, neuropsychological, neuroimaging and pathophysiological features. Prog Neurobiol 98, 99-143.

40. Nobuhara, K., Okugawa, G., Sugimoto, T., Minami, T., Tamagaki, C., Takase, K., Saito, Y., Sawada, S., Kinoshita, T., 2006. Frontal white matter anisotropy and symptom severity of late-life depression: a magnetic resonance diffusion tensor imaging study. J Neurol Neurosurg Psychiatry 77, 120-122.

41. Raffelt, D., Tournier, J.D., Rose, S., Ridgway, G.R., Henderson, R., Crozier, S., Salvado, O., Connelly, A., 2012. Apparent Fibre Density: a novel measure for the analysis of diffusion-weighted magnetic resonance images. Neuroimage 59, 39763994.

42. Raffelt, D.A., Smith, R.E., Ridgway, G.R., Tournier, J.D., Vaughan, D.N., Rose, S., Henderson, R., Connelly, A., 2015. Connectivity-based fixel enhancement: Wholebrain statistical analysis of diffusion MRI measures in the presence of crossing fibres. Neuroimage 117, 40-55. 
43. Reppermund, S., Zhuang, L., Wen, W., Slavin, M.J., Trollor, J.N., Brodaty, H., Sachdev, P.S., 2014. White matter integrity and late-life depression in communitydwelling individuals: diffusion tensor imaging study using tract-based spatial statistics. Br J Psychiatry.

44. Sarrazin, S., d'Albis, M.A., McDonald, C., Linke, J., Wessa, M., Phillips, M., Delavest, M., Emsell, L., Versace, A., Almeida, J., Mangin, J.F., Poupon, C., Le Dudal, K., Daban, C., Hamdani, N., Leboyer, M., Houenou, J., 2015. Corpus callosum area in patients with bipolar disorder with and without psychotic features: an international multicentre study. J Psychiatry Neurosci 40, 352-359.

45. Scheck, S.M., Pannek, K., Raffelt, D.A., Fiori, S., Boyd, R.N., Rose, S.E., 2015. Structural connectivity of the anterior cingulate in children with unilateral cerebral palsy due to white matter lesions. Neuroimage Clin 9, 498-505.

46. Schmahmann J, Pandya D, 2006. Fiber Pathways of the Brain. Oxford University Press, New York.

47. Sexton, C.E., Allan, C.L., Le Masurier, M., McDermott, L.M., Kalu, U.G., Herrmann, L.L., Maurer, M., Bradley, K.M., Mackay, C.E., Ebmeier, K.P., 2012a. Magnetic resonance imaging in late-life depression: multimodal examination of network disruption. Arch Gen Psychiatry 69, 680-689.

48. Sexton, C.E., Allan, C.L., Mackay, C.E., Ebmeier, K.P., 2012b. White matter integrity within the corpus callosum differentiates late-life bipolar and unipolar depression. Bipolar Disord 14, 790-791.

49. Sheehan, D.V., Lecrubier, Y., Sheehan, K.H., Amorim, P., Janavs, J., Weiller, E., Hergueta, T., Baker, R., Dunbar, G.C., 1998. The Mini-International Neuropsychiatric Interview (M.I.N.I.): the development and validation of a structured diagnostic psychiatric interview for DSM-IV and ICD-10. J Clin Psychiatry 59 Suppl 20, 2233;quiz 34-57.

50. Sheline, Y.I., Price, J.L., Vaishnavi, S.N., Mintun, M.A., Barch, D.M., Epstein, A.A., Wilkins, C.H., Snyder, A.Z., Couture, L., Schechtman, K., McKinstry, R.C., 2008. Regional white matter hyperintensity burden in automated segmentation distinguishes late-life depressed subjects from comparison subjects matched for vascular risk factors. Am J Psychiatry 165, 524-532.

51. Shimony, J.S., Sheline, Y.I., D'Angelo, G., Epstein, A.A., Benzinger, T.L., Mintun, M.A., McKinstry, R.C., Snyder, A.Z., 2009. Diffuse microstructural abnormalities of normal-appearing white matter in late life depression: a diffusion tensor imaging study. Biol Psychiatry 66, 245-252.

52. Suzuki, Y., Hori, M., Kamiya, K., Fukunaga, I., Aoki, S., M, V.A.N.C., 2016. Estimation of the Mean Axon Diameter and Intra-axonal Space Volume Fraction of the Human Corpus Callosum: Diffusion q-space Imaging with Low q-values. Magn Reson Med Sci 15, 83-93.

53. Thomas, A.J., O'Brien, J.T., Davis, S., Ballard, C., Barber, R., Kalaria, R.N., Perry, R.H., 2002. Ischemic basis for deep white matter hyperintensities in major depression: a neuropathological study. Arch Gen Psychiatry 59, 785-792.

54. Tomasch, J., 1954. Size, distribution, and number of fibres in the human corpus callosum. Anat Rec 119, 119-135.

55. Tournier, J.D., Calamante, F., Connelly, A., 2007. Robust determination of the fibre orientation distribution in diffusion MRI: non-negativity constrained super-resolved spherical deconvolution. Neuroimage 35, 1459-1472. 
56. Tournier, J.D., Yeh, C.H., Calamante, F., Cho, K.H., Connelly, A., Lin, C.P., 2008. Resolving crossing fibres using constrained spherical deconvolution: validation using diffusion-weighted imaging phantom data. Neuroimage 42, 617-625.

57. Valkanova, V., Ebmeier, K.P., 2013. Vascular risk factors and depression in later life: a systematic review and meta-analysis. Biol Psychiatry 73, 406-413.

58. van Uden, I.W., Tuladhar, A.M., de Laat, K.F., van Norden, A.G., Norris, D.G., van Dijk, E.J., Tendolkar, I., de Leeuw, F.E., 2015. White matter integrity and depressive symptoms in cerebral small vessel disease: The RUN DMC study. Am J Geriatr Psychiatry 23, 525-535.

59. Vaughan, D., Raffelt, D., Tournier, J.D., Jackson, G., Connelly, A., 2013. Apparent fibre density shows tract-specific white matter changes in temporal lobe epilepsy., 21st Annual Meeting of International Society for Magnetic Resonance Imaging in Medicine, Salt Lake City, USA.

60. Vos, S.B., Jones, D.K., Viergever, M.A., Leemans, A., 2011. Partial volume effect as a hidden covariate in DTI analyses. Neuroimage 55, 1566-1576.

61. Walterfang, M., Yucel, M., Barton, S., Reutens, D.C., Wood, A.G., Chen, J., Lorenzetti, V., Velakoulis, D., Pantelis, C., Allen, N.B., 2009. Corpus callosum size and shape in individuals with current and past depression. J Affect Disord 115, 411420.

62. Wang, L., Leonards, C.O., Sterzer, P., Ebinger, M., 2014. White matter lesions and depression: a systematic review and meta-analysis. J Psychiatr Res 56, 56-64.

63. Wardlaw, J.M., Valdes Hernandez, M.C., Munoz-Maniega, S., 2015. What are white matter hyperintensities made of? Relevance to vascular cognitive impairment. J Am Heart Assoc 4, 001140.

64. Wen, M.C., Steffens, D.C., Chen, M.K., Zainal, N.H., 2014. Diffusion tensor imaging studies in late-life depression: systematic review and meta-analysis. Int J Geriatr Psychiatry 29, 1173-1184.

65. Witelson, S.F., 1989. Hand and sex differences in the isthmus and genu of the human corpus callosum. A postmortem morphological study. Brain 112 ( Pt 3), 799835.

66. Yamada, S., Takahashi, S., Ukai, S., Tsuji, T., Iwatani, J., Tsuda, K., Kita, A., Sakamoto, Y., Yamamoto, M., Terada, M., Shinosaki, K., 2015. Microstructural abnormalities in anterior callosal fibers and their relationship with cognitive function in major depressive disorder and bipolar disorder: a tract-specific analysis study. $\mathrm{J}$ Affect Disord 174, 542-548.

67. Yesavage, J.A., Brink, T.L., Rose, T.L., Lum, O., Huang, V., Adey, M., Leirer, V.O., 1982. Development and validation of a geriatric depression screening scale: a preliminary report. J Psychiatr Res 17, 37-49.

68. Zhang, Y., Brady, M., Smith, S., 2001. Segmentation of brain MR images through a hidden Markov random field model and the expectation-maximization algorithm. IEEE Trans Med Imaging 20, 45-57. 


\section{Table \& Figure Legends}

\section{Table 1.Characteristics of patient and control sample.}

Legend: Summary of patient and control participant characteristics, including demographic, clinical and imaging data. The p-values were calculated from t-tests for continuous variables (e.g. age, rating-scales, imaging parameters) and a chi-square tests for gender. Abbreviations: AFD = apparent fibre density, $F A=$ fractional anisotropy, $M M S E=$ mini-mental state examination, $\mathrm{RD}=$ radial diffusivity, $\mathrm{SD}=$ standard deviation, $\mathrm{WMH}=$ white matter hyperintensity.

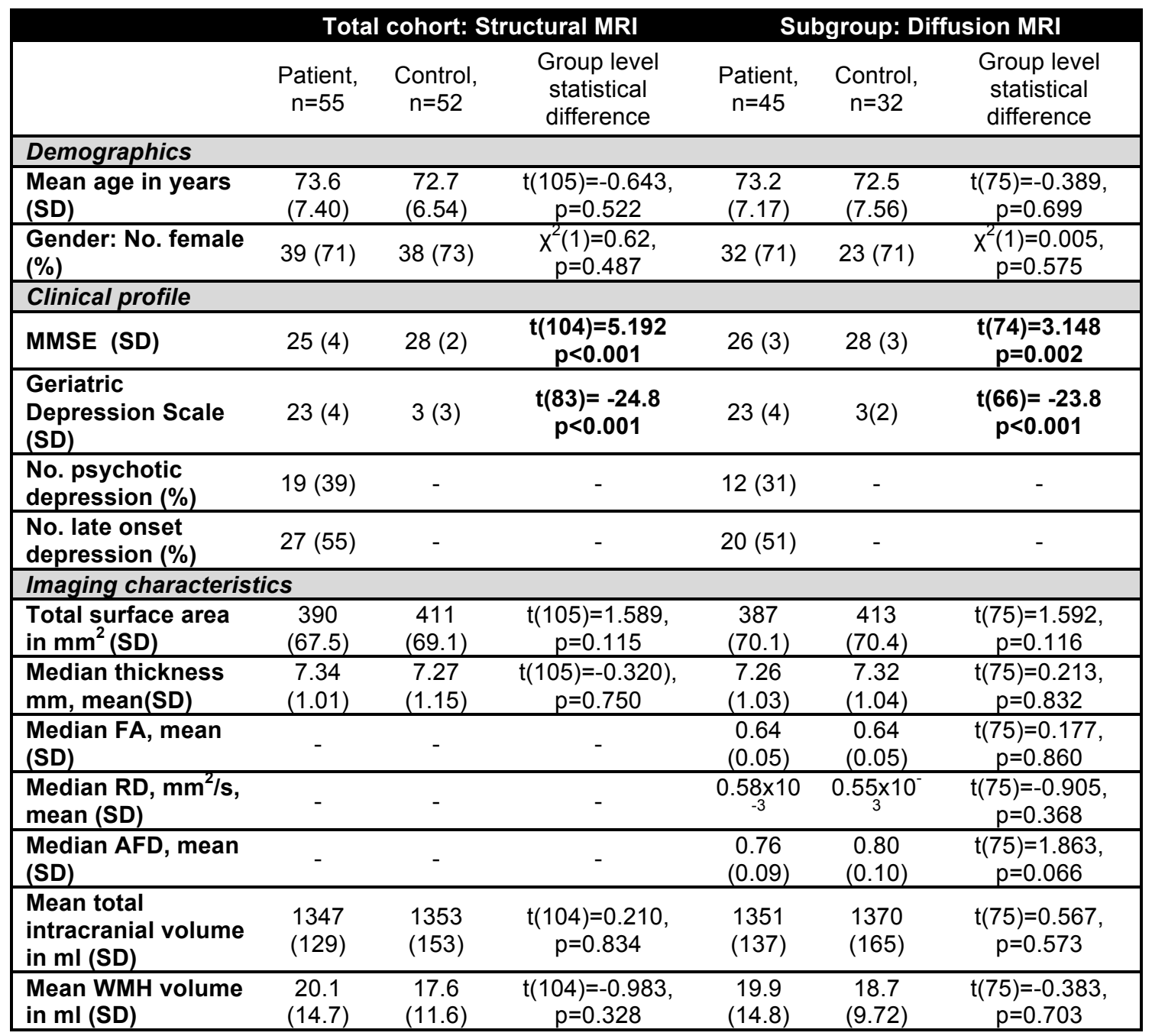


Table 2. Summary of patient versus control group differences in imaging parameters in

\section{the corpus callosum}

Legend: Summary of patient versus control group differences in imaging parameters in the whole corpus callosum and seven sub-regions. The $p$-values were calculated from ANCOVA models with diagnosis as a fixed factor, and age and total brain volume as covariates of nointerest. Additionally, potential scanner effects on the T1-weighted data were accounted for by including scanner as a fixed factor in the thickness and area analyses, and T2-effects were accounted for by including whole brain WMH volume as a covariate in the dMRI analyses. Abbreviations: $\mathrm{AFD}=$ apparent fibre density, $\mathrm{CC}=$ corpus callosum, $\mathrm{FA}=$ fractional anisotropy, $\eta^{2}=$ effect size, $R D=$ radial diffusivity.

\begin{tabular}{|c|c|c|c|c|c|}
\hline Region & Thickness & Area & $\mathrm{FA}$ & $\mathrm{RD}$ & AFD \\
\hline Total CC & $\begin{array}{l}F(1,105)=1.100 \\
p=0.297 \\
\eta^{2}=0.011\end{array}$ & $\begin{array}{l}F(1,105)=0.146 \\
p=0.703 \\
\eta^{2}=0.001\end{array}$ & $\begin{array}{l}F(1,75)=0.002 \\
p=0.963 \\
\eta^{2}<0.001\end{array}$ & $\begin{array}{l}F(1,75)=0.563 \\
p=0.456 \\
\eta^{2}=0.008\end{array}$ & $\begin{array}{l}F(1,75)=4.307 \\
p=0.042 \\
\eta^{2}=0.057\end{array}$ \\
\hline Genu & $\begin{array}{l}F(1,105)=2.031 \\
p=0.157 \\
\eta^{2}=0.020\end{array}$ & $\begin{array}{l}F(1,105)=1.063 \\
p=0.305 \\
\eta^{2}=0.010\end{array}$ & $\begin{array}{l}F(1,75)=2.417 \\
p=0.123 \\
\eta^{2}=0.033\end{array}$ & $\begin{array}{l}F(1,75)=0.364 \\
p=0.548 \\
\eta^{2}=0.005\end{array}$ & $\begin{array}{l}F(1,75)=1.465 \\
p=0.230 \\
\eta^{2}=0.020\end{array}$ \\
\hline Anterior body & $\begin{array}{l}F(1,105)=2.729 \\
p=0.102 \\
\eta^{2}=0.026\end{array}$ & $\begin{array}{l}F(1,105)=1.739 \\
p=1.90 \\
\eta^{2}=0.017\end{array}$ & $\begin{array}{l}F(1,75)=0.362 \\
p=0.549 \\
\eta^{2}=0.005\end{array}$ & $\begin{array}{l}F(1,75)=2.003 \\
p=0.161 \\
\eta^{2}=0.027\end{array}$ & $\begin{array}{l}F(1,75)=6.790 \\
p=0.011^{*} \\
\eta^{2}=0.087\end{array}$ \\
\hline Mid-body & $\begin{array}{l}F(1,105)=1.221 \\
p=0.272 \\
\eta^{2}=0.012\end{array}$ & $\begin{array}{l}F(1,105)=1.875 \\
p=0.174 \\
\eta^{2}=0.018\end{array}$ & $\begin{array}{l}F(1,75)=0.428 \\
p=0.515 \\
\eta^{2}=0.006\end{array}$ & $\begin{array}{l}F(1,75)=1.129 \\
p=0.292 \\
\eta^{2}=0.016\end{array}$ & $\begin{array}{l}F(1,75)=5.019 \\
p=0.028^{*} \\
\eta^{2}=0.066\end{array}$ \\
\hline Posterior body & $\begin{array}{l}F(1,105)=0.267 \\
p=0.606 \\
\eta^{2}=0.003\end{array}$ & $\begin{array}{l}F(1,105)=1.612 \\
p=0.207 \\
\eta^{2}=0.016\end{array}$ & $\begin{array}{l}F(1,75)=0.139 \\
p=0.710 \\
\eta^{2}=0.002\end{array}$ & $\begin{array}{l}F(1,75)=0.800 \\
p=0.374 \\
\eta^{2}=0.011\end{array}$ & $\begin{array}{l}F(1,75)=4.137 \\
p=0.046^{*} \\
\eta^{2}=0.055\end{array}$ \\
\hline Isthmus & $\begin{array}{l}F(1,105)=0.113 \\
p=0.737 \\
\eta^{2}=0.001\end{array}$ & $\begin{array}{l}F(1,105)=0.305 \\
p=0.582 \\
\eta^{2}=0.003\end{array}$ & $\begin{array}{l}\mathrm{F}(1,75)=0.001 \\
\mathrm{p}=0.974 \\
\eta^{2}<0.001\end{array}$ & $\begin{array}{l}F(1,75)=0.235 \\
p=0.629 \\
\eta^{2}=0.003\end{array}$ & $\begin{array}{l}F(1,75)=2.590 \\
p=0.112 \\
\eta^{2}=0.035\end{array}$ \\
\hline $\begin{array}{l}\text { Dorsal } \\
\text { splenium }\end{array}$ & $\begin{array}{l}F(1,105)=0.036 \\
p=0.850 \\
\eta^{2}<0.001\end{array}$ & $\begin{array}{l}F(1,105)=0.800 \\
p=0.373 \\
\eta^{2}=0.008\end{array}$ & $\begin{array}{l}F(1,75)=0.342 \\
p=0.560 \\
\eta^{2}=0.005\end{array}$ & $\begin{array}{l}F(1,75)=0.003 \\
p=0.954 \\
\eta^{2}<0.001\end{array}$ & $\begin{array}{l}\mathrm{F}(1,75)=2.171 \\
\mathrm{p}=0.145 \\
\eta^{2}=0.030\end{array}$ \\
\hline $\begin{array}{l}\text { Ventral } \\
\text { splenium }\end{array}$ & $\begin{array}{l}F(1,105)=0.329 \\
p=0.568 \\
\eta^{2}=0.003\end{array}$ & $\begin{array}{l}F(1,105)=0.173 \\
p=0.678 \\
\eta^{2}=0.002\end{array}$ & $\begin{array}{l}F(1,75)=2.475 \\
p=0.120 \\
\eta^{2}=0.034\end{array}$ & $\begin{array}{l}F(1,75)=2.407 \\
p=0.125 \\
\eta^{2}=0.033\end{array}$ & $\begin{array}{l}F(1,75)=4.873 \\
p=0.031^{*} \\
\eta^{2}=0.064\end{array}$ \\
\hline
\end{tabular}


Figure 1: Mid-sagittal corpus callosum parcellation scheme

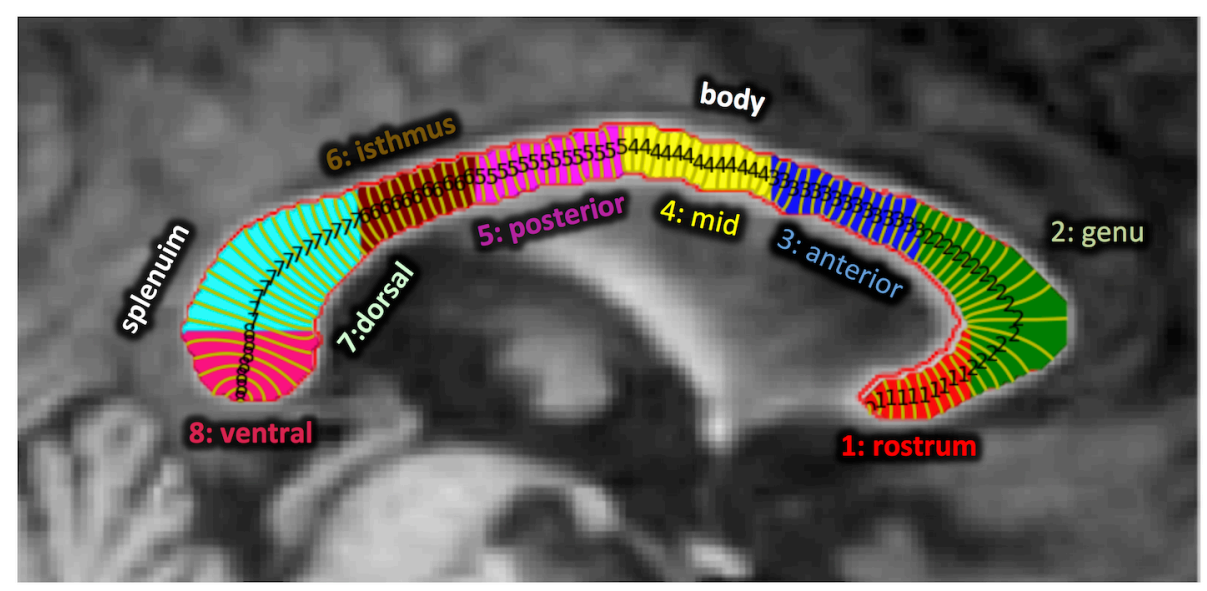

Legend: The corpus callosum mid-sagittal slice was defined in each subject's native T1 image using an automated pipeline and parcellated into 8 callosal sub-regions. Orientation: Sagittal view, left=posterior, right=anterior

Figure 2: Differences in apparent fibre density between patients and controls

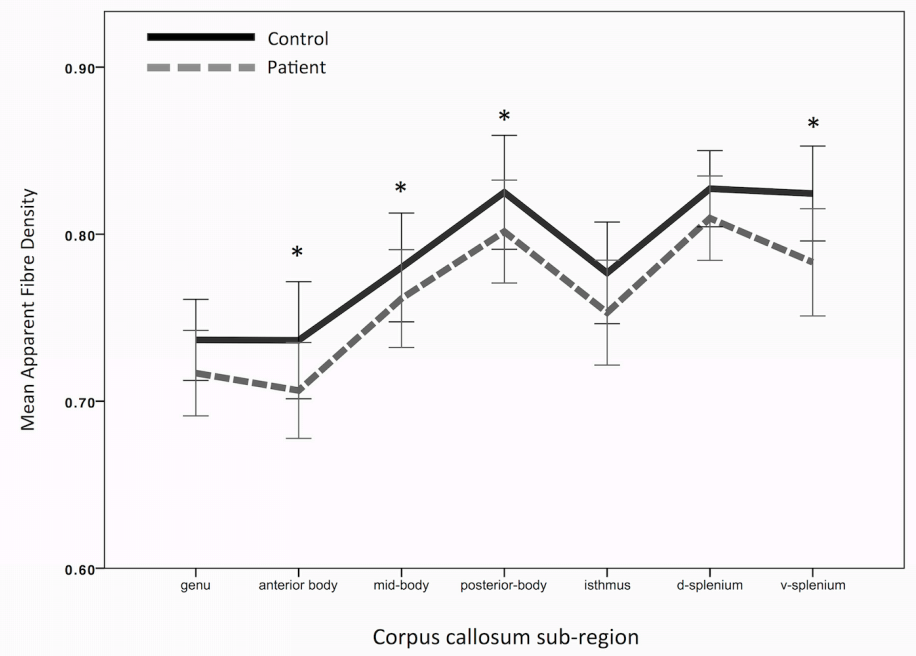

Legend: Plot of mean apparent fibre density in patients and controls in each callosal subregion. Error bars represent $95 \%$ confidence intervals. * denotes $p<0.05$ obtained from ANCOVA model with diagnosis as a fixed factor, and age, total brain volume and white matter hyperintensity volume as covariates. 